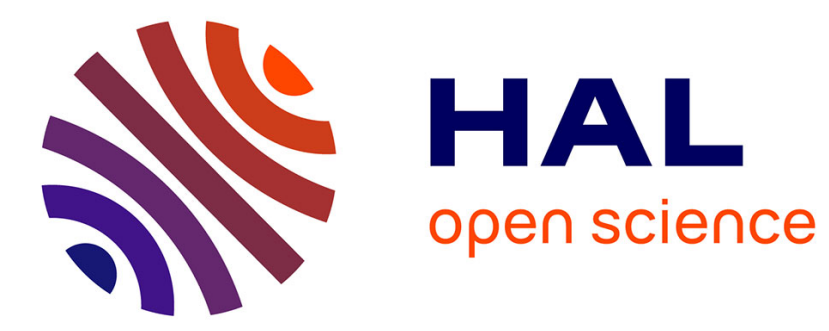

\title{
Multi-objective resources allocation approaches for workflow applications in Cloud environments
}

Kahina Bessai, Samir Youcef, Ammar Oulamara, Claude Godart, Selmin Nurcan

\section{- To cite this version:}

Kahina Bessai, Samir Youcef, Ammar Oulamara, Claude Godart, Selmin Nurcan. Multi-objective resources allocation approaches for workflow applications in Cloud environments. OnTheMove Federated Conferences \& Workshops, Sep 2012, Rome, Italy. hal-00751216

\author{
HAL Id: hal-00751216 \\ https://hal.inria.fr/hal-00751216
}

Submitted on 13 Nov 2012

HAL is a multi-disciplinary open access archive for the deposit and dissemination of scientific research documents, whether they are published or not. The documents may come from teaching and research institutions in France or abroad, or from public or private research centers.
L'archive ouverte pluridisciplinaire HAL, est destinée au dépôt et à la diffusion de documents scientifiques de niveau recherche, publiés ou non, émanant des établissements d'enseignement et de recherche français ou étrangers, des laboratoires publics ou privés. 


\title{
Multi-objective resources allocation approaches for workflow applications in Cloud environments
}

\author{
Kahina Bessai ${ }^{1}$, Samir Youcef $^{2}$, Ammar Oulamara $^{2}$, Claude Godart ${ }^{2}$ \\ and Selmin Nurcan ${ }^{1}$ \\ ${ }^{1}$ University of Paris1-Panthéon- Sorbonne, Centre de Recherche en Informatique \\ 90, rue de Tolbiac 75634 Paris, France \\ \{kahina.bessai, selmin.nurcan\}@malix.univ-paris1.fr \\ ${ }^{2}$ University of Lorraine, LORIA-INRIA-UMR 7503 \\ BP 239, Vandoeuvre-les-Nancy, France \\ \{youcef, oulamara, godart\}@loria.fr
}

\begin{abstract}
Resources allocation and scheduling has been recognised as an important topic for business process execution. However, despite the proven benefits of using Cloud to run business process, users lack guidance for choosing between multiple offering while taking into account several objectives which are often conflicting. Moreover, when running business processes it is difficult to automate all tasks. In this paper, we propose three complementary approaches for Cloud computing platform taking into account these specifications.
\end{abstract}

\section{Introduction}

The Cloud computing has quickly changed the way that compute resources can be used and allow users to access compute on the fly according to the application's need. For example, to run any desired software Amazon's EC2 provide a Web service through which users can boot an Amazon Machine Image. However, despite the proven benefits of using Cloud to execute business processes, users lack guidance for choosing between different offering while taking into account several objectives often conflicting.

Moreover, most existing workflow matching and scheduling algorithms consider only an environment in which the number of resources is assumed to be bounded. However, in distributed systems such as Cloud computing this assumption is in opposition to the usefulness of such systems. Indeed, the "illusion of infinite resources" is the most important feature of Clouds [2][3], which means that users can request, and are likely to obtain, sufficient resources for their need at any time. Additionally to this characteristic, a Cloud computing environment can provide several advantages that are distinct from other computing environments [3]. Moreover, unlike scientific workflows, where generally their processing are fully automated, when executing workflow processes it is difficult to automate all theirs tasks. Indeed, certain tasks require validations that cannot be automated because they are subject to human intervention.

To overcome the limitations of existing approaches for workflow process optimization, we propose an extension of our recent study [1]. 


\section{Problem formulation}

Definition 1 (Business process). A business process application is represented as a directed acyclic graph $(D A G)$ denoted $G=(T, E)$, where:

1. $T=\left\{t_{1}, \ldots, t_{n}\right\}$ is a finite set of tasks.

2. E represents the set of directed edges. An edge $\left(t_{i}, t_{j}\right)$ of graph $G$ corresponds to the data dependencies between these tasks (the data generated by $t_{i}$ is consumed by $t_{j}$ ).

3. Task $t_{i}$ is called the immediate parent of $t_{j}$ which is the immediate child task of $t_{i}$.

Let Data be a $n \times n$ matrix of communication data, where data $[i, j]$ is the amount of data required to be transmitted from task $t_{i}$ to task $t_{j}$.

Definition 2 (Resource graph). The resources are represented as a directed graph denoted $R G$. Formally, a resources graph is represented by $R G=(R, V)$, where:

1. $R=\left\{V M_{1}, \ldots, V M_{m}, H R_{1}, \ldots, H R_{m^{\prime}}\right\}$ is a finite set of virtual machines types and human resources.

2. $V$ represents the set of directed edges. Each edge is denoted $\left(V M_{i}, V M_{j}\right)$ corresponding to the link between these virtual machines.

Let $B$ be a $m \times m$ matrix, in which $B[i, j]$ is the bandwidth between virtual machine types $V M_{i}$ and $V M_{j}$, where $B[i, i] \longrightarrow \infty$ means that there is no transfer data.

Let $r\left(t_{j}\right)$ denotes the resource (virtual machine or human resource) that executes task $t_{j}$. The transfer time $T T\left(r\left(t_{i}\right), r\left(t_{j}\right)\right)$, which is for transferring data from task $t_{i}$ (executed by $\left.r\left(t_{i}\right)\right)$ to task $t_{j}$ (executed by $r\left(t_{j}\right)$ ) is defined by:

$$
T T\left(r\left(t_{i}\right), r\left(t_{j}\right)\right)=\frac{\operatorname{data}[i, j]}{B\left[r\left(t_{i}\right), r\left(t_{j}\right)\right]}
$$

Let $E T$ be a $n \times m$ execution time matrix in which $E T\left(t_{i}, r_{j}\right)$ gives the execution time estimation to complete task $t_{i}$ by resource $r_{j}$.

Let $U E C$ be a $\left(m+m^{\prime}\right)$-dimensional unit execution cost vector, where $U E C\left(r_{j}\right)$ represents the cost per time unit incurred by using the resource $r_{j}$. Let $E C$ be a $n \times m+m^{\prime}$ execution cost matrix in which $E C\left(t_{i}, r_{j}\right)$ gives the execution cost to complete task $t_{i}$ by resource $r_{j}$ defined by:

$$
E C\left(t_{i}, r_{j}\right)=E T\left(t_{i}, r_{j}\right) \times U E C\left(r_{j}\right)
$$

The data transfer cost $T C\left(r\left(t_{i}\right), r\left(t_{j}\right)\right)$, which is the cost incurred due to the transfer of data from task $t_{i}$ (executed by $\left.r\left(t_{i}\right)\right)$ to task $t_{j}$ (executed by $r\left(t_{j}\right)$ ), is defined by: $T C\left(r\left(t_{i}\right), r\left(t_{j}\right)\right)=$ data $[i, j] \times\left(C_{\text {out }}\left(r\left(t_{i}\right)\right)+C_{\text {in }}\left(r\left(t_{j}\right)\right)\right)$

where $C_{\text {out }}\left(r\left(t_{i}\right)\right)$ and $C_{i n}\left(r\left(t_{j}\right)\right)$ represent respectively the cost of transferring data from $r\left(t_{i}\right)$ and the cost of receiving data on $r\left(t_{j}\right)$. 


\section{The objective functions}

a. Time objective function Let EST (earliest start time) and EFT (earliest finish time) attributes that characterize the set of resources (virtual machine types and human resources). These attributes are derived from a given partial matching and scheduling (i.e. a task $t_{i}$ is assigned to virtual machine $V M\left(t_{i}\right)$ or human resource $H M\left(t_{i}\right)$ ). The partial schedule refers to the fact that for each task the earliest start time and the earliest finish time values are obtained using only the tasks that must be performed before it. $E S T\left(t_{i}, r_{j}\right)$ and $E F T\left(t_{i}, r_{j}\right)$ are the earliest execution start time and the earliest execution finish time of task $t_{i}$ on resource $r_{j}$, respectively. For the input task $t_{i n p u t}$, the earliest execution start time and the earliest execution finish time are given by Equation 1 and Equation 2, respectively:

$$
\begin{gathered}
E S T\left(t_{\text {input }}, r_{j}\right)=0 \\
\operatorname{EFT}\left(t_{\text {input }}, r_{j}\right)=\operatorname{ET}\left(t_{i}, r_{j}\right)
\end{gathered}
$$

For the other tasks in the graph, the EST and the EFT values are computed recursively, starting from the initial task, as shown in Equation 3 and Equation 4. In order to compute the EFT of a task $t_{j}$, all immediate predecessor tasks of $t_{j}$ must have been assigned and scheduled with the consideration of the transfer time.

$$
E F T\left(t_{i}, r_{j}\right)=E S T\left(t_{i}, r_{j}\right)+E T\left(t_{i}, r_{j}\right)
$$

$$
\operatorname{EST}\left(t_{i}, r_{j}\right)=\max \left\{\operatorname{avail}[j], \max _{t_{p} \in \operatorname{pred}\left(t_{j}\right)}\left[A F T\left(t_{p}\right)+T T\left(r\left(t_{p}\right), r\left(t_{i}\right)\right)\right]\right\}
$$

where $\operatorname{pred}\left(t_{i}\right)$ is the set of immediate predecessors of task $t_{i}$. avail $[j]$ is the earliest time at which resource $r_{j}$ is ready for task execution. As the number of virtual machines is assumed to be infinite, then avail $[j]=0$ for all the used virtual machines. In other words, if task $t_{i}$ is performed by resource $r_{j}$ which is a virtual machine then $\operatorname{EST}\left(t_{i}, r_{j}\right)$ is computed as follows:

$$
E S T\left(t_{i}, r_{j}\right)=\max _{t_{p} \in \operatorname{pred}\left(t_{j}\right)}\left\{A F T\left(t_{p}\right)+T T\left(r\left(t_{p}\right), r\left(t_{i}\right)\right)\right\}
$$

The avail $[j]$ is the time that resource $r_{j}$ completed the execution of the task $t_{i}$ and it is ready to execute another task. The inner max block in the EST equation returns the ready time, i.e. the time when all required data by $t_{i}$ has arrived at resource $r_{j}$.

After a task $t_{i}$ is scheduled on a resource $r_{j}$, the earliest start time and the earliest finish time of $t_{i}$ on resource $r_{j}$ is equal to the actual start time, denoted $A S T\left(t_{i}\right)$, and the actual finish time, denoted $A F T\left(t_{i}\right)$, of task $t_{i}$, respectively.

After all tasks in a graph are scheduled, the schedule length (i.e., the overall completion time) will be the actual finish time of the exit task (i.e. $A F T\left(t_{\text {exit }}\right)$ ). The schedule length, also called makespan, is defined as:

$$
\text { makespan }=A F T\left(t_{\text {exit }}\right)
$$


Predictive heuristic for human resource availabilities The actual finish time as computed previously does not take into account the fact that human resources can perform other tasks that do not belong to the same process. More precisely, the work list of a given human resource may contain work items of different processes. The realism of this assumption can be disputed as a human resources are "shared" by more than one process. Thus, it is might be desirable to design a procedure in order to predict the human resources availabilities. Concretely, we propose to estimate this availability values taking into account the previous observation. Let availability $[j]_{k+1}$ is the estimation of resource $j$ availability and $t_{i}$ the next task that can be performed by this resource. Instead of simply adapting to the computed availability using equation 4 , one can try to forecast and estimate what availability $[j]_{k+1}$ will be using the historical data.

b. Cost objective function The cost function is a structure independent criterion defined as the sum of the costs of executing all workflow tasks, given by:

$$
\cos t=\sum_{j=1}^{n}\left\{E C\left(r\left(t_{j}\right)\right)+\sum_{p \in \operatorname{pred}\left(t_{j}\right)} T C\left(r\left(t_{j}\right), r\left(t_{p}\right)\right)\right\}
$$

Thus, the cost objective function is to determinate the assignment of tasks of a given workflow application such that its overall execution cost is minimized.

To take into account these objectives simultaneously, we propose to use the proposed approaches in our study [1] by replacing the execution time and the execution cost by respectively Equations 4 and 7.

\section{Conclusion}

In this paper, we have proposed a model for business processes execution in Cloud computing environments. The proposed approaches extend our previous algorithms. More precisely, three complementary approaches are designed to deal with the problem of matching and scheduling business process tasks in Cloud context taking into account two objectives (execution time and cost incurred using a set of resources). We plan to extend the proposed work to take into account others criteria like carbon emission and energy cost.

\section{References}

1. K. Bessai, S. Youcef, A. Oulamara, C. Godart and S. Nurcan Bi-criteria workflow tasks allocation and scheduling in Cloud computing environments, In Cloud Computing 2012, pp. 638-645..

2. Workflow Management Coalition: Terminology \& Glossary, WfMC-TC-1011, 1999.

3. R. Buyya, S. Pandey and C. Vecchiola, Cloudbus toolkit for market-oriented cloud computing, In CloudCom'09: Proceedings of the 1st International Conference on Cloud Computing, volume 5931 of LNCS, pp. 24-44, Springer, Germany, December 2009. 